Article

\title{
Analysis of Salivary Glands and Saliva from Aedes albopictus and Aedes aegypti Infected with Chikungunya Viruses
}

\author{
Irma Sanchez-Vargas ${ }^{1}$, Laura C. Harrington ${ }^{2}{ }^{\mathbb{D}}$, William C. Black IV ${ }^{1}$ and Ken E. Olson ${ }^{1, *}$ \\ 1 Arthropod-borne and Infectious Diseases Laboratory, Colorado State University, Ft. Collins, CO 80523, USA; \\ Irma.Sanchez-Vargas@colostate.edu (I.S.-V.); william.black@colostate.edu (W.C.B.IV) \\ 2 Department of Entomology, Cornell University, Ithaca, NY 14850, USA; 1ch27@cornell.edu \\ * Correspondence: Kenneth.olson@colostate.edu
}

Received: 18 December 2018; Accepted: 19 January 2019; Published: 1 February 2019

check for updates

\begin{abstract}
Chikungunya virus (CHIKV) is a medically important mosquito-borne virus transmitted to humans by infected Aedes (Stegomyia) species. In 2013-2014, Ae. aegypti transmitted CHIKV to humans in the Caribbean and in 2005-2006, Ae. albopictus transmitted CHIKV on La Réunion Island (Indian Ocean basin). CHIKV LR2006 OPY1 from the La Réunion epidemic was associated with a mutation (E1:A226V) in the viral E1 glycoprotein that enhanced CHIKV transmission by Ae. albopictus. CHIKV R99659 from the Caribbean outbreak did not have the E1:A226V mutation. Here, we analyzed the salivary glands and saliva of Ae. albopictus strains from New Jersey, Florida, Louisiana and La Réunion after infection with each virus to determine their transmission potential. We infected the Ae. albopictus strains with blood meals containing 3-7 $\times 10^{7} \mathrm{PFU} / \mathrm{mL}$ of each virus and analyzed the mosquitoes nine days later to maximize infection of their salivary glands. All four Ae. albopictus strains were highly susceptible to LR2006 OPY1 and R99659 viruses and their CHIKV disseminated infection rates (DIR) were statistically similar $(p=0.3916$ ). The transmission efficiency rate (TER) was significantly lower for R99659 virus compared to LR2006 OPY1 virus in all Ae. albopictus strains and Ae. aegypti (Poza Rica) $(p=0.012)$ suggesting a salivary gland exit barrier to R99659 virus not seen with LR2006 OPY1 infections. If introduced, LR2006 OPY1 virus poses an increased risk of transmission by both Aedes species in the western hemisphere.
\end{abstract}

Keywords: chikungunya virus; arbovirus; Aedes albopictus; Aedes aegypti; salivary glands; and saliva; salivary gland exit barrier

\section{Introduction}

Chikungunya virus (CHIKV; Alphavirus; Togaviridae) is an arthropod-borne virus (arbovirus) maintained during epidemics in transmission cycles between viremic humans and infected Aedes (Stegomyia) mosquitoes. The genome of CHIKV is a positive single stranded RNA ( 11,800 nucleotides) containing two open reading frames (ORFs) between the $5^{\prime}$ and $3^{\prime}$ untranslated regions [1]. The first ORF, encodes the nonstructural polyprotein (nsP1-nsP4), and the second ORF encodes the structural proteins (C, E3, E2, 6K and E1). The two ORFS are separated by a small untranslated junction region [1]. CHIK disease in symptomatic patients presents as fever and intense rash, weakness, myalgia and polyarthralgia [2]. The onset of fever coincides with viremia, and the viral load can rapidly reach up to $10^{9}$ viral genome copies per milliliter of blood or virus titers of $10^{7-9}$ plaque-forming unit equivalents $/ \mathrm{mL}[2,3]$. The principle vector of CHIKV is Ae. aegypti, a peridomestic, day-biting mosquito found in tropical and subtropical regions of the world [2]. However, in 2005-2006 an outbreak of CHIKV occurred on La Réunion Island (Indian Ocean basin) that was transmitted by 
another day biting mosquito, Ae. albopictus [4]. Significantly, Ae. albopictus has invaded new regions globally. They now thrive in temperate areas of the western hemisphere and could pose additional health risks to a CHIKV susceptible human population [5].

CHIKV are classified in three genotypic groups: East-Central-South Africa (ECSA), West Africa, and Asian. After the 2006 La Réunion outbreak, a new Indian Ocean lineage (IOL) was identified as a monophyletic descendant of the ECSA group [6-8]. The RNA genomes of ECSA-IOL CHIKV were associated with a genetic mutation in the CHIKV E1 glycoprotein-coding region resulting in a single alanine to valine amino acid change (E1-A226V) that adapted the virus to Ae. albopictus [9-12]. Others have reported that ECSA-IOL CHIKV infection of Ae. albopictus virus can be further enhanced by amino acid changes in their E2 glycoprotein [13]. In 2013-2014, CHIKV (Asian genotype) spread to more than 22 countries in the western hemisphere and caused tens of thousands of human cases [14-16]. The R99659 CHIKV was isolated from the British Virgin Islands during an epidemic but CHIKVs of the ECSA-IOL genotype were not associated with this outbreak [15]. However, the ECSA-IOL CHIKV could rapidly spread in the western hemisphere if viremic individuals with the ECSA-IOL CHIKV genotype travel to the United States (U.S.) and infect established Ae. albopictus populations. Autochthonous transmission of ECSA-IOL CHIKV by Ae. albopictus could then lead to new outbreaks in CHIKV-naïve human populations [17].

Our goal was to analyze salivary gland and the titer of virus in saliva of the two CHIKV isolates, LR2006 OPY1 (ECSA-IOL genotype) and R99659 (Asian genotype), in strains of Ae. albopictus from New Jersey, Florida and Louisiana. We compared infections of these mosquitoes with an Ae. albopictus strain from La Réunion Island and an Ae. aegypti strain from Mexico (Poza Rica). The extrinsic incubation period (EIP), the time from initial acquisition of CHIKV by the mosquito until virus transmission ranges from 2 to 9 days [18]. We analyzed salivary gland and saliva infection status for CHIKV in each infected mosquito at 9 days post-blood meal infection. Typically, CHIKV infects the midgut when the mosquito imbibes a viremic blood meal. The virus then disseminates to other tissues including salivary glands where the virus enters saliva. Virus in saliva is transmitted to a susceptible host during acquisition of a subsequent blood meal. To evaluate dissemination and transmission rates, saliva was collected to determine transmission potential, and then salivary glands were dissected from the same mosquitoes to analyze virus dissemination. Here, we report dissemination rate (DIR) as the percent of total mosquitoes exposed to virus that were positive for virus in the salivary gland and the transmission efficiency rate (TER) as the percent of mosquitoes positive for virus in the saliva that were also positive for virus in the salivary glands. Vector competence was determined here by multiplying DIR $\times$ TER. We clearly showed under laboratory conditions that the Louisiana (Lake Charles), Florida, and New Jersey strains of Ae. albopictus had high DIR values. The TER values for CHIKVs were lower than expected for R99659 virus given the mosquitoes' high DIRs and suggested the presence of a salivary gland escape barrier (SGEB) for that virus. All mosquitoes were competent to transmit LR2006 OPY1. Of the three U.S. Ae. albopictus strains, the Louisiana and Florida strains could serve as excellent vectors for CHIKV R99569 (Asian) transmission. The New Jersey Ae. albopictus strain had the lowest vector competence (DIR $\times$ TER) of the three Ae. albopictus strains for R99569 virus but had statistically similar vector competence as the other strains for LR2006 OPY1 (ECSA-IOL) [19].

\section{Materials and Methods}

\subsection{Virus and Cell Culture}

Vero cells, LLC-MK2 monkey kidney cells and C6/36 (A. albopictus) cells were cultured in modified Eagle's medium (MEM) supplemented with $8 \%$ fetal bovine serum, L-glutamine, non-essential amino acids and penicillin/streptomycin and maintained at $5 \% \mathrm{CO}_{2}$ at $37^{\circ} \mathrm{C}$ and $28^{\circ} \mathrm{C}$, respectively. The two low passage CHIKV isolates (R99659, British Virgin Islands, Asian genotype; Accession \# KJ451624.1) and LR2006 OPY1 (La Réunion, ECSA genotype; Accession \# DQ443544.2) were provided by the Centers for Diseases Control and Prevention (CDC-Fort Collins, CO, USA). The presence of the E1-A226V 
mutation in LR2006 OPY1 virus was confirmed by RT-PCR using specific primer sets designed to amplify the nucleotide sequence surrounding the mutation as previously described [20]. We infected Vero cells with low passage CHIKV at a 0.001 multiplicity of infection (MOI) then incubated cells at $37^{\circ} \mathrm{C}$ for $72 \mathrm{~h}$. Virus aliquots were stored at $-80{ }^{\circ} \mathrm{C}$ until used for preparing infectious blood meals.

\subsection{Mosquitoes}

Aedes albopictus from Florida, Louisiana (Lake Charles), and La Réunion Island (Indian Ocean) were provided by the Centers for Diseases Control and Prevention (CDC-Fort Collins, CO, USA). The New Jersey Ae. albopictus strain was obtained from Cornell University and the Ae. aegypti line was collected in Poza Rica, Mexico [4,21]. The La Réunion, New Jersey and Poza Rica mosquitoes were less than 10 generations in colony, Florida and Louisiana strains were more generations in colony. Mosquito eggs were hatched to obtain the immature stages and eclosed adults were held in cages at $28 \pm 1{ }^{\circ} \mathrm{C}$ and $70-80 \%$ relative humidity. Adults were fed 10\% sucrose ad libitum and maintained at 16:8 light:dark photoperiod. Groups of 200 adult females (1 week post-eclosion) were placed in $2.5 \mathrm{~L}$ cartons, deprived of sugar and water overnight to promote feeding of artificial blood meals consisting of virus-infected Vero cell and medium suspension (60\% vol/vol), 40\% (vol/vol) defibrinated sheep blood (Colorado Serum Co., Boulder, CO, USA) and $1 \mathrm{mM}$ ATP [22]. Blood engorged mosquitoes were maintained at the indicated insectary conditions for 9 days. Virus titers (pfu/mL) in the blood meal were quantified using a plaque assay described below.

\subsection{Infectious Virus Titration by Plaque Assay}

Plaque assays were performed using confluent monolayers of Vero cells in 24-well plates to determine virus titers in saliva and salivary glands. The samples were sterilized by passing them through Acrodisc HT Tuffryn 0.2- $\mu \mathrm{m}$ syringe filters (Pall Life Sciences, East Hills, NY, USA). Vero cells were infected for $1 \mathrm{~h}$ with 10-fold serial dilutions of saliva or salivary gland homogenate samples. Infected cells were overlaid with a $1 \%$ agar-nutrient mixture (agar solution $\left(1 \mathrm{~g} / 77 \mathrm{~mL}\right.$ DI $\left.\mathrm{H}_{2} \mathrm{O}\right)$ : nutrient solution $(10 \mathrm{~mL}$ of $10 \times / 100 \mathrm{~mL}$ Media 199, 7\% heat inactivated fetal bovine serum (FBS), 7.5\% sodium bicarbonate (4mL/100mL), 2\% DEAE- dextrose in Hanks balanced solution $(1 \mathrm{~mL} / 100 \mathrm{~mL}), 0.5 \mathrm{~mL} / 100 \mathrm{~mL}$ MEM essential amino acids $\left(15 \times\right.$ solution), and $0.5 \mathrm{~mL} / 100 \mathrm{~mL}$ MEM vitamins $\left(100 \times\right.$ solution)). After 7 days incubation at $37^{\circ} \mathrm{C}$ cells were stained with $3 \mathrm{mg} / \mathrm{mL}$ MTT (3-(4,5-dimethylthiazol-2-yl)-2,5-diphenyltetrazolium bromide) solution and incubated for $4 \mathrm{~h}[23,24]$. Viral titers were determined by counting visible plaques and individual saliva or salivary gland titers were reported as $\mathrm{pfu} / \mathrm{mL}$.

\subsection{Saliva Collection and Salivary Gland Dissections}

At day 9 post-infections, saliva from each female was collected using a previously described saliva collection method [25]. Briefly, females were chilled, and their wings and legs removed. The proboscis of the mosquito was inserted into a $1.0 \mu \mathrm{L}$ micropipette (microcaps, Drummond Scientific Company, Broomall, PA, USA) filled with immersion oil type B and allowed to salivate into the oil at room temperature. After 30-45 min, the proboscis was removed from the capillary and oil containing the saliva was expelled under pressure into $1.5 \mathrm{~mL}$ tubes containing $300 \mu \mathrm{L}$ DMEM medium $(20 \%$ of heat inactivated FBS, 1\% penicillin/streptomycin, 1\% glutamine, 1\% non-essential amino acids) and frozen immediately on dry ice. We also measured salivation volume from mosquitoes by allowing them to expectorate into $1 \mu \mathrm{L}$ capillary tubes as described previously and measured the height of the saliva with a digital fractional caliper (presicion: $\pm 0.02 \mathrm{~mm}$ ). The volume of saliva was calculated using the cylinder volume formula $\left(\mathrm{V}=\pi\left(\mathrm{r}^{2} \times \mathrm{h}\right)=3.1416 \times 0.01 \times \mathrm{h}=\mathrm{mm}^{3}\right.$ or $\left.\mu \mathrm{L}\right)$. Following expectoration, salivary glands were dissected from the same mosquitoes that provided saliva and placed in $500 \mu \mathrm{L}$ of DMEM medium (7\% of heat inactivated FBS, 1\% penicillin/streptomycin, $1 \%$ glutamine, $1 \%$ non-essential amino acids). The salivary gland samples were frozen immediately (dry ice) prior to determining CHIKV titers. CHIKV titers in saliva and salivary glands were reported as $\mathrm{pfu} / \mathrm{mL}$ from plaque assays as previously described. 


\subsection{Statistical Analyses}

The proportion of infected salivary glands and saliva were calculated by averaging the number of infected samples divided by the total tested. All data were analyzed with GraphPad prism software (version 5.0, La Jolla, CA, USA) for testing significant differences $(p<0.05)$ in DIR and TER (Fisher's exact test, $\chi^{2}$ test) and the correlation among virus titers in saliva and salivary glands (Pearson correlation). Analysis of variance (one-way and two-way ANOVA) was used to determine the statistical significance and CORREL function (Excel 2016, Microsoft, Redmond, DC, USA) for determining the correlation coefficient.

\section{Results}

\subsection{Dissemination of CHIKV R99659 and LR2006 OPY1 to Salivary Glands of Ae. albopictus Mosquito Strains}

The DIR of Ae. albopictus for CHIKV was evaluated 9 days after infection with R99659 (blood meal virus titer: $5.6 \times 10^{7} \mathrm{pfu} / \mathrm{mL}$ ) or LR2006 OPY1 (blood meal virus titer: $6.6 \times 10^{7} \mathrm{pfu} / \mathrm{mL}$ ). We detected no significant difference in DIR among the Ae. albopictus strains (Florida, Louisiana, New Jersey, and La Réunion) infected with either R99659 and LR2006 OPY1 ( $p=0.3916)$. DIR ranged from $93 \%$ to $96.6 \%$ for R99659 and $93.3 \%$ to $100 \%$ for LR2006 OPY1. However, significant differences were detected in the virus titers of salivary glands among the Ae. albopictus strains infected with either R99659 $(p<0.0001)$ or LR2006 OPY1 ( $p=0.0461$; Figure 1). R99659 virus titers in the salivary glands of the La Réunion strain were significantly higher than titers in the New Jersey strain $(p=0.0010)$ or Louisiana strain $(p=0.0181)$. LR2006 OPY1 virus titers in the New Jersey strain were significantly lower than in the Florida and La Réunion strains of Ae. albopictus ( $p=0.0330$ and $p=0.0282$, respectively; Figure 1). While the LR2006 OPY1 virus titers were higher than R99659 virus titers in the salivary glands of all Ae. albopictus strains tested, statistically significant differences were detected between R99659 and LR2006 OPY1 virus titers in the La Réunion $(p=0.0453)$, Florida $(p=0.0089)$ and New Jersey $(p=0.0472)$ strains. We observed no significant differences in DIR between the two CHIKV isolates in the Louisiana strain $(p=0.1920)$.

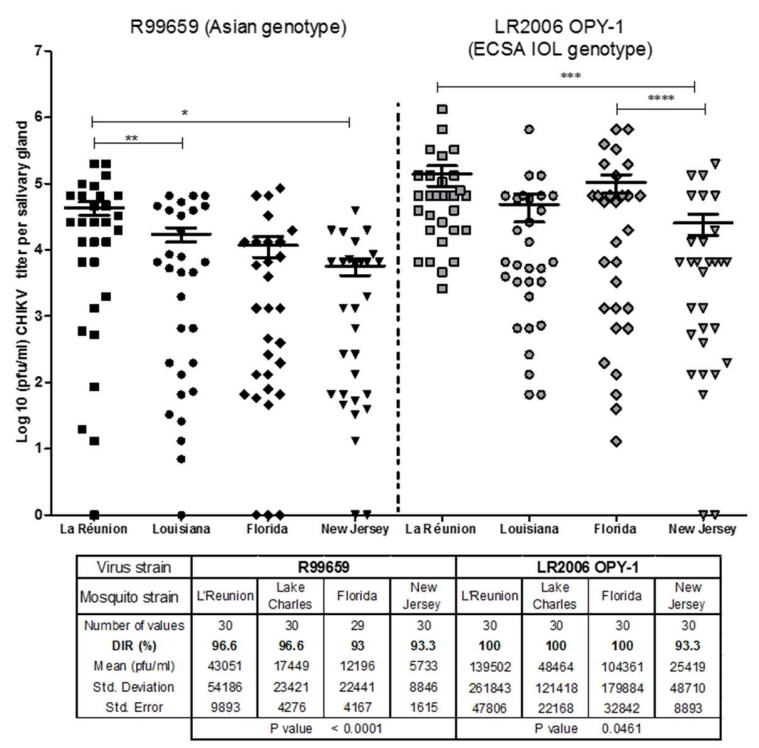

Figure 1. Chikungunya virus (CHIKV) disseminated infection rates (DIR) and titer in salivary glands of four Ae. albopictus strains. Aedes albopictus were orally infected with either CHIKV R99659 (blood titer $5.6 \times 10^{7} \mathrm{pfu} / \mathrm{mL}$ ) or CHIKV LR2006 OPY1 (blood titer $6.6 \times 10^{7} \mathrm{pfu} / \mathrm{mL}$ ) and the DIR (prevalence) and virus titers were determined 9 days post infection in salivary glands by plaque assay. The number of mosquitoes assayed from each mosquito and CHIKV strain and the prevalence (\% infection) are listed. Horizontal bars show mean titer values with standard error of the mean (SEM). $p$-values of virus titers among the Ae. albopictus strains infected with either R99659 or LR2006 OPY1 are shown in table. ${ }^{*} p=0.0010,{ }^{* *} p=0.0181,{ }^{* * *} p=0.0330,{ }^{* * * *} p=0.0282$. 


\subsection{CHIKV R99659 and LR2006 OPY1 Viruses in Saliva of Ae. albopictus Mosquito Strains}

We did not detect significant differences in virus prevalence in saliva (TER) among the Ae. albopictus strains infected with R99659 virus ( $p=0.3916)$ or the Ae. albopictus strains infected with LR2006 OPY1 virus. The prevalence (TER) of virus in saliva ranged from $33.3 \%$ to 56.6\% for R99659 virus and $80 \%$ to $86.6 \%$ for CHIKV LR2006 OPY1 virus. The TER of R99659-infected Ae. albopictus strains was significantly lower than mosquito strains infected with LR2006 OPY1 virus $(p<0.0001$; Figure 2). Significant differences were also observed for virus titers in saliva among Ae. albopictus strains infected with R99659 ( $p=0.012$. Figure 2). No significant differences were observed for LR2006 OPY1 ( $p=0.7544$. Figure 2) titers in saliva of the four Ae. albopictus strains. The LR2006 OPY1 titers in saliva of the New Jersey strain were significantly higher than R99659 titers in saliva of the same strain $(p<0.0001)$ possibly indicating LR2006 OPY1 virus has a fitness advantage in these mosquitoes.

R99659 (Asian genotype)

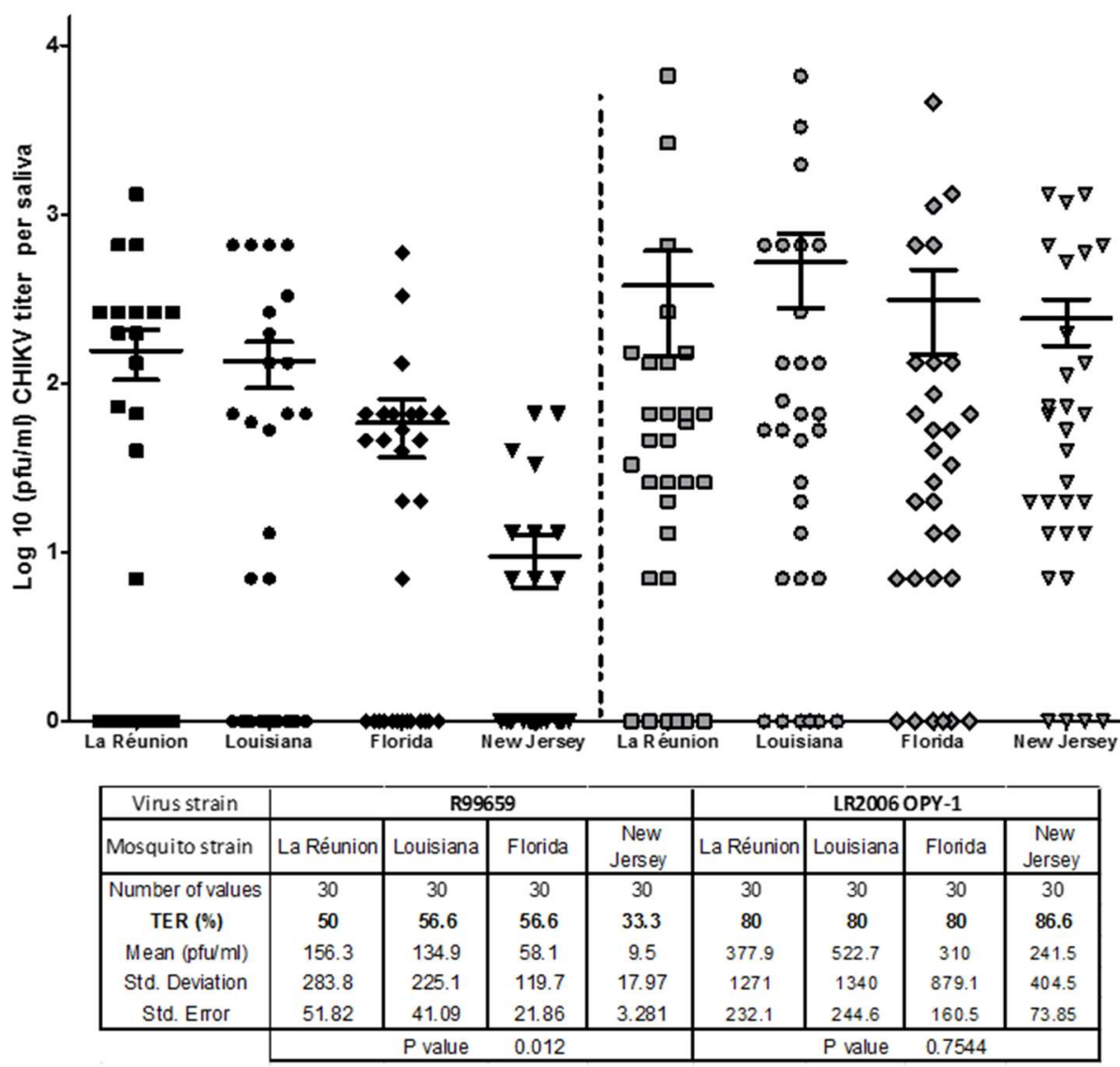

Figure 2. Transmission efficiency rate (TER) assay of CHIKVs in four Ae. albopictus strains. The TER (prevalence) and virus titer were determined for Ae. albopictus (three U.S. strains and the La Réunion strain) orally infected with either CHIKV R99659 (blood meal virus titer $5.6 \times 10^{7} \mathrm{pfu} / \mathrm{mL}$ ) or CHIKV LR2006 OPY1 (blood meal virus titer $6.6 \times 10^{7} \mathrm{pfu} / \mathrm{mL}$ ). Virus titers were determined 9 days later in saliva by plaque assay. Saliva for each female was collected using forced saliva capillary method. The number of mosquitoes assayed from each mosquito and CHIKV strain and the prevalence (\% infection) are listed. Horizontal bars show mean titer values with SEM. $p$ value of virus titers among the Ae. albopictus strains infected with either R99659 or LR2006 OPY1 were $p=0.012$ and $p=0.7544$, respectively. 
3.3. Dissemination and Transmission Potential of CHIKV R99659 and LR2006 OPY1 in Ae. aegypti (Poza Rica)

The DIRs of $A$. aegypti (Poza Rica) for R99659 (blood meal virus titer $6.2 \times 10^{7} \mathrm{pfu} / \mathrm{mL}$ ) or LR2006 OPY1 (blood meal virus titer $6.6 \times 10^{7} \mathrm{pfu} / \mathrm{mL}$ ) were also evaluated 9 days post-infection. DIRs were high for all CHIKV isolates tested in Ae. aegypti, with no significant difference $(p=0.3679)$ between R99659 and LR2006 OPY1 viruses (Figure 3). The DIRs were 92\% for R99659 and 93\% for LR2006 OPY1 viruses (Figure 3). The TER for Ae. aegypti (Poza Rica) infected with LR2006 OPY1 and R99659 viruses were statistically similar $(p>0.05)$. These data indicated that Ae. aegypti (Poza Rica) strains had statistically similar vector competence (DIR $\times$ TER) for these viruses. DIR, TER and vector competence for the two CHIKVs and the four Ae. albopictus strains and one Ae. aegypti strain are summarized in Table 1.

Table 1. DIR, TER, and vector competence for R99659 and LR2006 OPY1 CHIKVs and the four Ae. albopictus strains and one Ae. aegypti strain. $n=30$ for all pairwise test.

\begin{tabular}{cccc}
\hline \multicolumn{4}{c}{ R99659 CHIKV R99659 } \\
\hline Ae. albopictus & $\begin{array}{c}\text { Dissemination Rates } \\
\text { (DIR) }\end{array}$ & $\begin{array}{c}\text { Transmission Efficiency } \\
\text { Rate (TER) }\end{array}$ & $\begin{array}{c}\text { Vector Competence } \\
\text { (VC) }\end{array}$ \\
\hline La Réunion & 96.6 & 50 & 48.3 \\
Louisiana & 96.6 & 56.6 & 54.7 \\
Florida & 93 & 56.6 & 52.7 \\
New Jersey & 93.3 & 33.3 & 31.1 \\
\hline \multicolumn{4}{c}{ LR2006 OPY1 CHIKVOPY1 ZIKVOPY-1 } \\
\hline Ae. albopictus & Dissemination Rates & Transmission Efficiency & Vector Competence \\
& (DIR) & Rate (TER) & 80 \\
\hline La Réunion & 100 & 80 & 80 \\
Louisiana & 100 & 80 & 80 \\
Florida & 100 & 80 & 80.1 \\
New Jersey & 93.3 & 86.6 & \\
\hline & \multicolumn{2}{c}{ Aedes aegypti (Poza Rica) } \\
\hline ZIKV & Dissemination Rates & Transmission Efficiency & Vector Competence \\
& (DIR) & Rate (TER) & (VC) \\
\hline R99659 & 92 & 50 & 46 \\
\hline
\end{tabular}




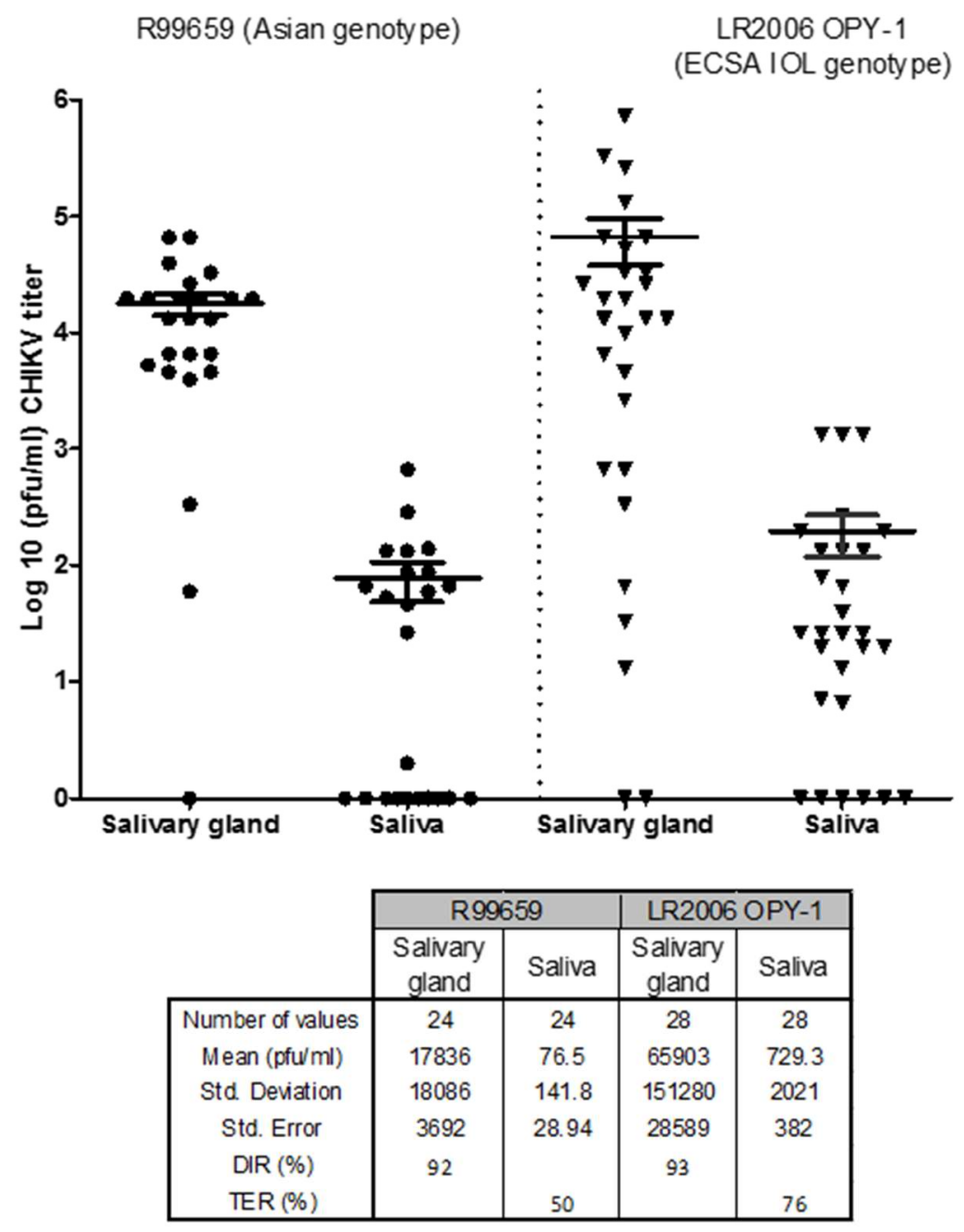

Figure 3. CHIKV titers in Poza Rica A. aegypti strain from Mexico. Ae. aegypti (Poza Rica) were orally infected with either CHIKV R99659 (blood meal virus titer $6 \times 10^{7} \mathrm{pfu} / \mathrm{mL}$ ) and CHIKV LR2006 OPY1 (blood meal virus titer $3 \times 10^{7} \mathrm{pfu} / \mathrm{mL}$ ). Virus titers were determined 9 days later by plaque assay in salivary glands and saliva. The number of mosquitoes assayed for each CHIKV strain and the prevalence (\% infection) of CHIKV in saliva are listed. Horizontal bars show mean titer values with SEM.

\subsection{Relationship between CHIKV Infectious Particles in Saliva and Salivary Glands}

We observed no correlation between salivary gland virus titer $(\mathrm{pfu} / \mathrm{mL}$ ) and saliva virus titer (pfu/mL) for R99659 and LR2006 OPY1 viruses in Ae. aegypti (Poza Rica; Figure 4A). This was also true for the four Ae. albopictus strains infected with R99659 genotype (Figure 4B) and the Florida and Louisiana Ae. albopictus strains infected with LR2006 OPY1 (Figure 4C). However, the TER of the La Réunion Ae. albopictus strain infected with LR2006 OPY1 showed a positive correlation between salivary gland virus titer and saliva virus titer (correlation coefficient $=0.672$ ). Although the New Jersey A. albopictus strain had a positive correlation between the two variables, it was weak and likely insignificant (correlation coefficient $=0.323$; Figure $4 \mathrm{C}$ ). 
A.

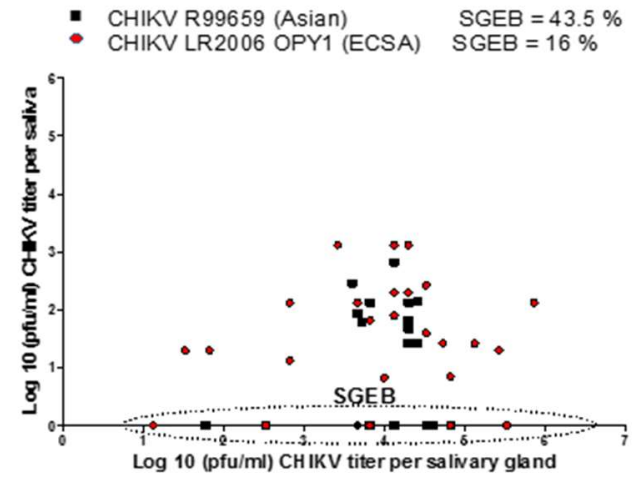

B.

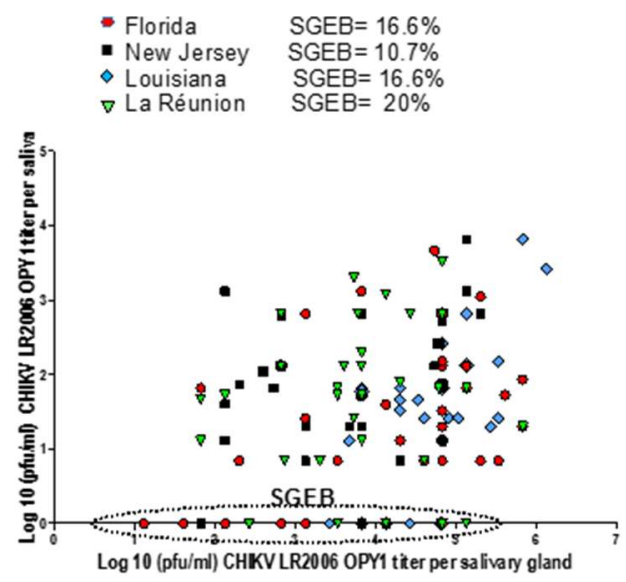

C.

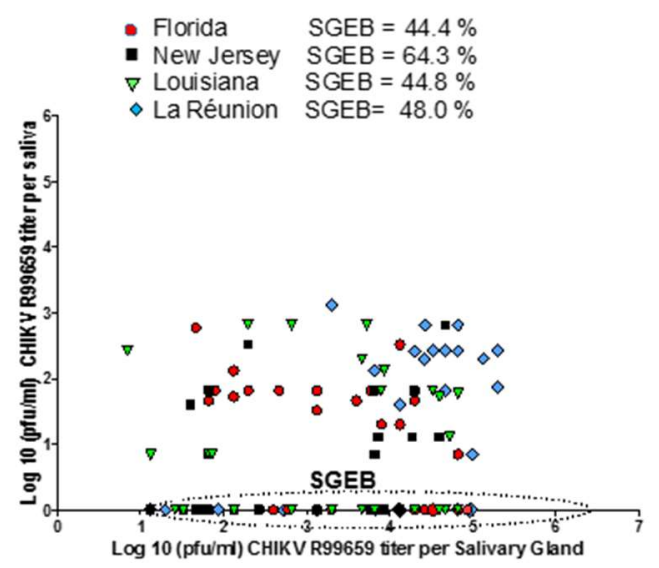

Figure 4. Correlation between CHIKV titers of saliva and salivary glands. The presence of a salivary gland escape barrier (SGEB) was tested by correlating CHIKV titers in salivary glands with virus titers in saliva. Saliva was collected at 9 days post infection and then salivary glands were dissected from the same mosquitoes. Virus titers from salivary glands and saliva were determined by plaque assays. (A) Correlation between saliva and salivary gland of Ae. aegypti (Poza Rica) infected with CHIKV R99659 (•) and CHIKV LR2006 OPY1 (ם). (B) Correlation between saliva and salivary gland of Ae. albopictus infected with CHIKV R99659. Florida strain (•), New Jersey strain (ם), Louisiana, $(\nabla)$ and La Réunion $(\diamond)$. (C) Correlation between saliva and salivary gland of Ae. albopictus infected with CHIKV LR2006 OPY1. Florida strain $(\bullet)$, New Jersey strain $(\boldsymbol{\square})$, Louisiana, $(\nabla)$ and La Réunion $(\diamond)$. The \% SGEB is the number of saliva samples without virus divided by the number of virus positive salivary gland samples. Percentage of mosquitoes with SGEB are listed for each group.

The presence of a salivary gland escape barrier (SGEB) was determined by dividing the number of saliva samples without virus by the number of virus positive salivary glands. The highest SGEB occurred with the Ae. aegypti (Poza Rica) strain since many mosquitoes were without detectable virus in their saliva even though their salivary glands were highly infected and all mosquitoes were able to salivate regardless of their infection status. This was also true for the four Ae. albopictus strains tested but the correlation was weaker than the SGEBs associated with Ae. aegypti. Interestingly, SGEB was dependent upon the viral genotype, since the four Ae. albopictus strains and the Ae. aegypti strain infected with R99659 (Asian genotype) showed a higher SGEB than mosquitoes infected with LR2006 OPY1 virus. The New Jersey Ae. albopictus strain infected with R99659 virus presented the highest SGEB (64.3\%) compared with the Florida, La Réunion or Ae. aegypti (Poza Rica) strains (SGEB $=44.4 \%, 44.8 \%, 48 \%$ and $43.5 \%$ respectively; Figure $4 \mathrm{~A}-\mathrm{C}$ ). In contrast, only $10.7 \%$ to $20 \%$ of mosquitoes presented a SGEB with LR2006 OPY1 (ECSA IOL genotype), however, the titer of virus in the salivary glands did vary significantly between mosquitoes infected with R99659 and LR2006 OPY1. 
3.5. Vector Competence of Ae. aegypti (Poza Rica) and Ae. albopictus Strains for R99659 versus LR2006 OPY1 Viruses

We multiplied DIR by TER to determine vector competence of each group of mosquitoes ( $n=30$ /group) for each virus. The four Ae. albopictus strains had significantly higher vector competence for LR2006 OPY1 virus versus R99659 virus ( $p=0.0001$; Table 1). Ae. aegypti (Poza Rica) also had a significantly higher vector competence for LR2006 OPY1 virus than R99659 virus $(p=0.005)$. Comparisons of the vector competence of Ae. aegypti versus Ae. albopictus for LR2006 OPY1 were not different $(p=0.1881)$. Comparisons of Ae. aegypti (Poza Rica) versus the Ae. albopictus strains (La Réunion, Louisiana, and Florida) for R99659 were also not statistically different $(p>0.05)$. The only significant differences when comparing vector competence of mosquito groups for R99659 virus was between the New Jersey strain and the Florida or Louisiana Ae. albopictus groups $(p=0.0191$ and $p=0.0083)$.

\subsection{Measurement of Salivation Using Ae. aegypti as A Model for Determining Saliva Volume from An Aedes Species}

In our assay, saliva is expectorated just after the mosquito proboscis immerses into collection fluid. Mosquitoes such as Culex. pipiens salivate an average of $4.7 \mathrm{~nL}$ during the blood feeding process [26]. The rate of salivation may vary depending on the species, age, size, physiological state of the mosquito and environmental conditions. We initially determined the average of saliva volume produced during probing by uninfected Ae. aegypti. Here we used a capillary tube $(0.2 \mathrm{~mm}$ inner diameter) prefilled with $1 \mu \mathrm{L}$ of immersion oil type B. Twenty-six mosquitoes were allowed to salivate into the oil at room temperature for $1 \mathrm{~h}$ and then measured the height of saliva in the capillary tube with a digital fractional caliper (presicion: $\pm 0.02 \mathrm{~mm}$ ). The volume of saliva was then calculated using the cylinder volume formula $\left(\mathrm{V}=\pi\left(\mathrm{r}^{2} \times \mathrm{h}\right)=3.1416 \times 0.01 \times \mathrm{h}=\mathrm{mm}^{3}\right.$ or $\mu \mathrm{L}$; Figure $\left.5 \mathrm{~A}\right)$. After creating the frequency distribution (histogram) and "Gaussian" distribution using GraphPad Prism software, we calculated that Ae. aegypti mosquitoes salivated an average of $6.82 \pm 2.88 \mathrm{~nL}$ (Figure 5B). We then determined whether the volume of saliva influenced virus titer by collecting the saliva in calibrated capillary tubes and quantifying the expectorate volume. The titer of CHIKV (pfu/mL) did not strongly correlate with the volume of saliva, since volumes $2-3 \mathrm{~nL}$ of saliva contained as much or more virus titer than 5-7 nL volumes of saliva (Figure 5C). Figure $5 \mathrm{C}$ is one data set from multiple collections of saliva and determinations of CHIKV titer. We have observed little correlation between virus titer and saliva volume for other arboviruses such as Zika and dengue-2 viruses (Flavivirus; unpublished data) from hundreds of Ae. aegypti saliva samples [27]. 
A.

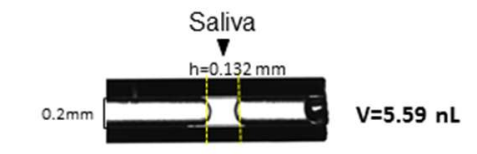

Saliva volume $=\pi\left(\mathrm{r}^{2} \times \mathrm{h}\right)=3.1416 \times .01 \times \mathrm{h}=\mathrm{mm} \mathrm{m}^{3}=\mu \mathrm{L}$

B.

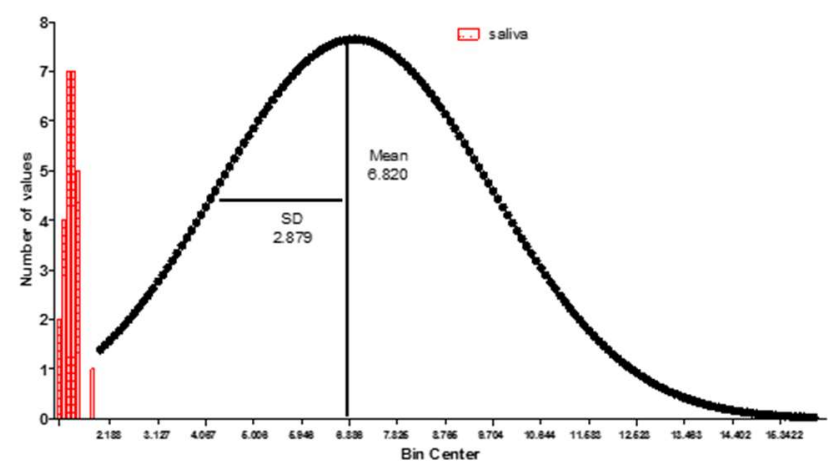

C.

\begin{tabular}{ccc}
\hline $\begin{array}{c}\text { Salivary } \\
\text { gland titer }\end{array}$ & $\begin{array}{c}\text { Saliva } \\
\text { titer }\end{array}$ & $\begin{array}{c}\text { Saliva } \\
\text { Volumen }\end{array}$ \\
\hline 66000 & 79.2 & $7.1 \mathrm{n} \mathrm{L}$ \\
198000 & 145.2 & $\square$ \\
13200 & 13.2 & \\
66000 & 0 & \\
264000 & 660 & \\
26400 & 132 & \\
198000 & 1320 & \\
264000 & 1320 & \\
66000 & 79.2 & \\
33000 & 13.2 & \\
66000 & 132 & \\
33000 & 0 & $1.88 \mathrm{~nL}$ \\
\hline
\end{tabular}

Figure 5. Frequency and Gaussian distribution of saliva volume in Ae. aegypti. The average saliva volume produced by Ae. aegypti, was determined by forcing mosquitoes to salivate into a calibrated capillary tube $(0.2 \mathrm{~mm}$ inner diameter) filled with immersion oil type B. Twenty six mosquitoes were allowed to salivate into the oil at room temperature for $1 \mathrm{~h}$. (A) An image of a capillary with saliva was captured after the mosquito expectorated. The height of saliva was measured using a digital fractional caliper (presicion: $\pm 0.02 \mathrm{~mm}$ ). The volume of saliva was calculated using the cylinder volume formula $\left(\mathrm{V}=\pi\left(\mathrm{r}^{2} \times \mathrm{h}\right)=3.1416 \times 0.01 \times \mathrm{h}=\mathrm{mm}^{3}\right.$ or $\left.\mu \mathrm{L}\right)$. (B) Frequency distribution (histogram) and Gaussian distribution using GraphPad Prism software. The mean value at the center of the distribution and SD is a measure of the width of the distribution. Aedes mosquitoes salivated an average of $6.82 \pm 2.88 \mathrm{~nL}$. (C) $R 99659$ titers were determined from a range of saliva volumes between 1.8 and $7 \mathrm{~nL}$. We did not see a strong correlation between virus titers in salivary gland and the volume of saliva. Arrow indicates saliva descending volumes between $7.1 \mathrm{~nL}$ and $1.88 \mathrm{~nL}$.

\section{Discussion}

Vector competence, the ability of an arbovirus vector to acquire a virus and successfully transmit the virus to another susceptible host, is determined by genetic traits of vector species and vector populations within species. Vector competence, the efficiency of viral replication and external factors including temperature, the availability of vertebrate hosts, vector population density and predation, determine how successful a vector population will be in transmitting virus in nature [28]. DIR and TER analyses of laboratory-infected Ae. albopictus and Ae. aegypti populations were performed here to indicate vector competence. We reasoned that the high DIR values ranging from $93 \%$ to $100 \%$ of infected salivary glands for each Aedes species lessened the importance of analyzing midgut infection rates 9 days after per os infection.

In our study, the Ae. albopictus strains clearly had uniformly high DIR or CHIKV prevalence in salivary glands after infection with R99659 or LR2006 OPY1 virus. Although the DIRs were similar among the Ae. albopictus strains tested, the salivary glands infected with the two CHIKVs varied in virus titers. The mean virus titer of both CHIKVs were highest for the La Réunion strain and lowest for the New Jersey strain indicating that geographic origin of the vector is an important factor. Nevertheless, differences in vector competence of the New Jersey strain for LR2006 OPY1 were not significant when compared to the other Ae. albopictus strains and Ae. aegypti (Poza Rica) (see Table 1). CHIKV studies to date have demonstrated distinct differences in vector competence of Ae. aegypti and Ae. albopictus depending on geographic origin of the mosquitoes and the genotype of CHIKV [28-33]. 
Although the Ae. albopictus strains had statistically similar DIRs for both CHIKVs, the TER for CHIKV R99659 virus was significantly lower in all mosquito strains (Ae. albopictus and Ae. aegypti) than the LR2006 OPY1 virus ( $p=0.012$ ) suggesting the LR2006 OPY1 virus is more efficiently transmitted than R99659 virus. The New Jersey strain had lower TER values than the other Ae. albopictus strains tested suggesting that population was less able to transmit CHIKV. Our results support earlier reports that noted similar DIR and TER outcomes for CHIKVs in Ae. aegypti and Ae. albopictus populations originating in the Americas [32].

We found no correlation between the virus titer in the salivary gland and virus titers in the saliva (Figure 4) and demonstrated in our Ae. aegypti model that the volume of saliva does not influence virus titers in saliva (Figure 5). Although we have not measured saliva volume from Ae. albopictus or correlated their saliva volume with virus titer, this will be a topic of future experiments. CHIKV provides evidence of how an arbovirus can acquire the capacity for efficient transmission by a new vector mosquito species with unfortunate medical outcomes. During the CHIKV's emergence in the Indian Ocean basin, it acquired a mutation in the coding sequence of the envelope glycoprotein E1 that resulted in the substitution of a valine for an alanine at position 226 (A226V) of E1 [12]. CHIKV with this mutation increased the vector competence of Ae. albopictus for LR2006 OPY1 [10]. Interestingly, the CHIKV that was introduced into the Caribbean in 2013 lacked the A226V mutation, and this mutation has not yet been detected in CHIKVs from the Americas [34]. Given that arboviruses are subject to antiviral pressures from the mosquito, arboviral genome diversity in infected mosquito vectors likely allowed selection of CHIKV-IOL that were better adapted to Ae. albopictus to ensure the virus' survival and transmission [35].

The DIR and TER data suggests that the salivary glands of Aedes vectors may lead to barriers to CHIKV transmission that vary with mosquito and viral genetics. Our data demonstrated that 93-100\% of mosquitoes had salivary gland infections but only $33-86 \%$ of saliva samples were positive for the two CHIKVs. This difference was more pronounced in Ae. aegypti than Ae. albopictus. We also measured saliva volume from $A$ e. aegypti and saw little correlation between the volume of expectorate and virus titer. SGEBs have been reported for La Crosse (LACV) and Sindbis virus transmission by Aedes and Culex species, respectively [36-39]. For example, Aedes hendersoni was shown to be an incompetent vector of LACV due to a SGEB in which the salivary glands were infected but the mosquito failed to transmit the virus orally [39]. More recently, a SGEB has been reported that affects Rift Valley Fever virus transmission [40]. Molecular mechanisms that explain SGEB for arboviruses will probably include a combination of vector and viral genetic factors that determine the efficiency of arbovirus transmission from a vector population. Arbovirus infection of salivary glands typically begins in the distal lateral lobes [41-43]. Certain arboviruses such as CHIKV infect the proximal lateral and median lobes of Ae. aegypti [42] and following replication, virus deposits in the apical cavities of acinar cells, which can lead to inoculation of a susceptible host upon refeeding [44]. We demonstrated here that TER in Ae. aegypti and Ae. albopictus vary according to the origins of mosquito populations tested and the CHIKV strain.

CHIKV is now widespread worldwide and likely will continue to pose a public health threat globally wherever the invasive mosquitoes Ae. albopictus and Ae. aegypti exists to transmit CHIKV to a susceptible human population. The emergence of mosquito-borne CHIKV in the Americas starting in 2013 led to a geographically widespread outbreak with human illness and the establishment of CHIKV transmission cycles in urban settings. As evidence of this, 11 cases from autochthonous transmission of CHIK were detected in south Florida in 2014 [45] emphasizing the importance of knowing the relative vector competence of North American mosquitoes to this arbovirus. This knowledge, coupled with enhanced understanding of critical arbovirus-vector interactions and the ecology of the major vectors will enable locally specific, efficient deployment of public health resources in the event of another outbreak of CHIKV in North America. 


\section{Conclusions}

(1) We infected the Ae. albopictus strains and Ae. aegypti with blood meals containing 5-7 $\times 10^{7} \mathrm{PFU} / \mathrm{mL}$ of R99659 (Asian genotype) and LR2006 OPY1 (ECSA-IOL genotype) and analyzed the mosquitoes nine days later to detect maximum infection of their salivary glands and saliva.

(2) All four Ae. albopictus strains were highly susceptible to LR2006 OPY1 and R99659 viruses and their CHIKV disseminated infection rates (DIR) were statistically similar $(p=0.3916)$.

(3) We demonstrated here that the TER in Ae. aegypti and Ae. albopictus vary according to their geographic origins of the mosquitoes and the CHIKV strain contributing to a salivary gland barrier for releasing R99659 virus into saliva.

(4) Virus titers in salivary glands did not correlate with saliva volume, but Ae. aegypti expectorated on average a volume of $6.8 \mathrm{~nL} /$ mosquito.

(5) If introduced, LR2006 OPY1 virus could increase the risk of CHIKV transmission by both Aedes species in the western hemisphere.

Author Contributions: Conceptualization, I.S.-V., L.C.H., W.C.B.IV, and K.E.O.; Methodology, L.C.H.; Validation, K.E.O.; Formal Analysis, I.S.-V. and K.E.O.; Investigation, I.S.-V., L.C.H., W.C.B.IV, and K.E.O.; Data Curation, I.S.-V.; Writing-Original Draft Preparation, I.S.-V.; Writing-Review \& Editing, L.C.H., W.C.B.IV, and K.E.O.; Funding Acquisition, L.C.H. and W.C.B.IV.

Funding: This project was supported by the following grants: NIH R21-AI 128374-02 (I.S.-V., K.E.O., and W.C.B.), NIH/NIAID-R01AI095491 (L.C.H.), Centers for Disease Control and Prevention (U01 CK000509) (L.C.H.) and Cornell Affinito Stewart Sabbatical Grant (L.C.H.). The funders had no role in study design, data collection and analysis, decision to publish, or preparation of the manuscript.

Conflicts of Interest: The authors declare no conflict of interest.

\section{References}

1. Strauss, J.H.; Strauss, E.G. The alphaviruses: Gene expression, replication, and evolution. Microbiol. Rev. 1994, 58, 491-562. [PubMed]

2. Weaver, S.C.; Lecuit, M. Chikungunya virus and the global spread of a mosquito-borne disease. N. Engl. J. Med. 2015, 372, 1231-1239. [CrossRef]

3. Riswari, S.F.; Ma'roef, C.N.; Djauhari, H.; Kosasih, H.; Perkasa, A.; Yudhaputri, F.A.; Artika, I.M.; Williams, M.; van der Ven, A.; Myint, K.S.; et al. Study of viremic profile in febrile specimens of chikungunya in Bandung, Indonesia. J. Clin. Virol. 2016, 74, 61-65. [CrossRef] [PubMed]

4. Van Duijl-Richter, M.K.; Hoornweg, T.E.; Rodenhuis-Zybert, I.A.; Smit, J.M. Early events in chikungunya virus infection-from virus cell binding to membrane fusion. Viruses 2015, 7, 3647-3674. [CrossRef] [PubMed]

5. Rai, K.S. Aedes albopictus in the Americas. Annu. Rev. Entomol. 1991, 36, 459-484. [CrossRef]

6. Powers, A.M.; Brault, A.C.; Tesh, R.B.; Weaver, S.C. Re-emergence of Chikungunya and O'nyong-nyong viruses: Evidence for distinct geographical lineages and distant evolutionary relationships. J. Gen. Virol. 2000, 81 Pt 2, 471-479. [CrossRef]

7. Volk, S.M.; Chen, R.; Tsetsarkin, K.A.; Adams, A.P.; Garcia, T.I.; Sall, A.A.; Nasar, F.; Schuh, A.J.; Holmes, E.C.; Higgs, S.; et al. Genome-scale phylogenetic analyses of chikungunya virus reveal independent emergences of recent epidemics and various evolutionary rates. J. Virol. 2010, 84, 6497-6504. [CrossRef]

8. Dupont-Rouzeyrol, M.; Caro, V.; Guillaumot, L.; Vazeille, M.; D’Ortenzio, E.; Thiberge, J.M.; Baroux, N.; Gourinat, A.C.; Grandadam, M.; Failloux, A.B. Chikungunya virus and the mosquito vector Aedes aegypti in New Caledonia (South Pacific Region). Vector Borne Zoonotic Dis. 2012, 12, 1036-1041. [CrossRef]

9. Tsetsarkin, K.A.; Vanlandingham, D.L.; McGee, C.E.; Higgs, S. A single mutation in chikungunya virus affects vector specificity and epidemic potential. PLoS Pathog. 2007, 3, 0030201. [CrossRef] [PubMed]

10. Tsetsarkin, K.A.; McGee, C.E.; Volk, S.M.; Vanlandingham, D.L.; Weaver, S.C.; Higgs, S. Epistatic roles of E2 glycoprotein mutations in adaption of chikungunya virus to Aedes albopictus and Ae. aegypti mosquitoes. PLoS ONE 2009, 4, 0006835. [CrossRef] 
11. Vazeille, M.; Moutailler, S.; Coudrier, D.; Rousseaux, C.; Khun, H.; Huerre, M.; Thiria, J.; Dehecq, J.S.; Fontenille, D.; Schuffenecker, I.; et al. Two Chikungunya isolates from the outbreak of La Reunion (Indian Ocean) exhibit different patterns of infection in the mosquito, Aedes albopictus. PLoS ONE 2007, 2, 0001168. [CrossRef] [PubMed]

12. Schuffenecker, I.; Iteman, I.; Michault, A.; Murri, S.; Frangeul, L.; Vaney, M.C.; Lavenir, R.; Pardigon, N.; Reynes, J.M.; Pettinelli, F.; et al. Genome microevolution of chikungunya viruses causing the Indian Ocean outbreak. PLoS Med. 2006, 3, 23. [CrossRef] [PubMed]

13. Tsetsarkin, K.A.; Weaver, S.C. Sequential adaptive mutations enhance efficient vector switching by Chikungunya virus and its epidemic emergence. PLoS Pathog. 2011, 7, 8. [CrossRef] [PubMed]

14. Waggoner, J.J.; Pinsky, B.A. How great is the threat of chikungunya virus? Expert Rev. Anti Infect. Ther. 2015, 13, 291-293. [CrossRef] [PubMed]

15. Morrison, T.E. Reemergence of chikungunya virus. J. Virol. 2014, 88, 11644-11647. [CrossRef] [PubMed]

16. Silva, J.V.J., Jr.; Ludwig-Begall, L.F.; de Oliveira-Filho, E.F.; Oliveira, R.A.S.; Duraes-Carvalho, R.; Lopes, T.R.R.; Silva, D.E.; Gil, L.H. A scoping review of Chikungunya virus infection: Epidemiology, clinical characteristics, viral co-circulation complications, and control. Acta Trop. 2018, 6, 30937-30939. [CrossRef] [PubMed]

17. Gratz, N.G. Critical review of the vector status of Aedes albopictus. Med. Vet. Entomol. 2004, 18, $215-227$. [CrossRef] [PubMed]

18. Rudolph, K.E.; Lessler, J.; Moloney, R.M.; Kmush, B.; Cummings, D.A. Incubation periods of mosquito-borne viral infections: A systematic review. Am. J. Trop Med. Hyg. 2014, 90, 882-891. [CrossRef]

19. Vanlandingham, D.L.; Higgs, S.; Huang, Y.J. Aedes albopictus (Diptera: Culicidae) and mosquito-borne viruses in the United States. J. Med. Entomol. 2016, 53, 1024-1028. [CrossRef]

20. Thavara, U.; Tawatsin, A.; Pengsakul, T.; Bhakdeenuan, P.; Chanama, S.; Anantapreecha, S.; Molito, C.; Chompoosri, J.; Thammapalo, S.; Sawanpanyalert, P.; et al. Outbreak of chikungunya fever in Thailand and virus detection in field population of vector mosquitoes, Aedes aegypti (L.) and Aedes albopictus Skuse (Diptera: Culicidae). Southeast. Asian J. Trop Med. Public Health. 2009, 40, 951-962.

21. Vera-Maloof, F.Z.; Saavedra-Rodriguez, K.; Elizondo-Quiroga, A.E.; Lozano-Fuentes, S.; Black IV, W.C. Coevolution of the Ile1,016 and Cys1,534 mutations in the voltage gated sodium channel gene of Aedes aegypti in Mexico. PLoS Negl. Trop Dis. 2015, 9, e0004263. [CrossRef]

22. Bosio, C.F.; Beaty, B.J.; Black, W.C., IV. Quantitative genetics of vector competence for dengue-2 virus in Aedes aegypti. Am. J. Trop Med. Hyg. 1998, 59, 965-970. [CrossRef]

23. Sladowski, D.; Steer, S.J.; Clothier, R.H.; Balls, M. An improved MTT assay. J. Immunol. Methods. 1993, 157, 203-207. [CrossRef]

24. Takeuchi, H.; Baba, M.; Shigeta, S. An application of tetrazolium (MTT) colorimetric assay for the screening of anti-herpes simplex virus compounds. J. Virol. Methods. 1991, 33, 61-71. [CrossRef]

25. Dubrulle, M.; Mousson, L.; Moutailler, S.; Vazeille, M.; Failloux, A.B. Chikungunya virus and Aedes mosquitoes: Saliva is infectious as soon as two days after oral infection. PLoS ONE 2009, 4, 0005895. [CrossRef]

26. Hurlbut, H.S. Mosquito salivation and virus transmission. Am. J. Trop. Med. Hyg. 1966, 15, 989-993. [CrossRef] [PubMed]

27. Sanchez-Vargas, I.; Olson, K.E.; Black, W.C., IV. Genetics of salivary gland infection barriers in population of Aedes aegypti. Infect. Genet. Evol.. (In preparation)

28. Vega-Rua, A.; Zouache, K.; Girod, R.; Failloux, A.B.; Lourenco-de-Oliveira, R. High level of vector competence of Aedes aegypti and Aedes albopictus from ten American countries as a crucial factor in the spread of Chikungunya virus. J. Virol. 2014, 88, 6294-6306. [CrossRef] [PubMed]

29. Alto, B.W.; Wiggins, K.; Eastmond, B.; Velez, D.; Lounibos, L.P.; Lord, C.C. Transmission risk of two chikungunya lineages by invasive mosquito vectors from Florida and the Dominican Republic. PLoS Negl. Trop Dis. 2017, 11, e0005724. [CrossRef]

30. Honorio, N.A.; Wiggins, K.; Camara, D.C.P.; Eastmond, B.; Alto, B.W. Chikungunya virus vector competency of Brazilian and Florida mosquito vectors. PLoS Negl. Trop. Dis. 2018, 12, e0006521. [CrossRef]

31. Ngoagouni, C.; Kamgang, B.; Kazanji, M.; Paupy, C.; Nakoune, E. Potential of Aedes aegypti and Aedes albopictus populations in the Central African Republic to transmit enzootic chikungunya virus strains. Parasit Vectors. 2017, 10, 164. [CrossRef] 
32. Lounibos, L.P.; Kramer, L.D. Invasiveness of Aedes aegypti and Aedes albopictus and vectorial capacity for chikungunya virus. J. Infect. Dis. 2016, 214 (Suppl. 5), S453-S458. [CrossRef]

33. Reiskind, M.H.; Pesko, K.; Westbrook, C.J.; Mores, CN. Susceptibility of Florida mosquitoes to infection with chikungunya virus. Am. J. Trop. Med. Hyg. 2008, 78, 422-425. [CrossRef] [PubMed]

34. Ruckert, C.; Ebel, G.D. How do virus-mosquito interactions lead to viral emergence? Trends Parasitol. 2018, 34, 310-321. [CrossRef]

35. Forrester, N.L.; Coffey, L.L.; Weaver, S.C. Arboviral bottlenecks and challenges to maintaining diversity and fitness during mosquito transmission. Viruses 2014, 6, 3991-4004. [CrossRef] [PubMed]

36. Beaty, B.J.; Holterman, M.; Tabachnick, W.; Shope, R.E.; Rozhon, E.J.; Bishop, D.H. Molecular basis of bunyavirus transmission by mosquitoes: Role of the middle-sized RNA segment. Science 1981, 211, 1433-1435. [CrossRef] [PubMed]

37. Grimstad, P.R.; Paulson, S.L.; Craig, G.B., Jr. Vector competence of Aedes hendersoni (Diptera: Culicidae) for La Crosse virus and evidence of a salivary-gland escape barrier. J. Med. Entomol. 1985, 22, 447-453. [CrossRef]

38. Jupp, P.G. Culex theileri and Sindbis virus; salivary glands infection in relation to transmission. J. Am. Mosq. Control Assoc. 1985, 1, 374-376.

39. Paulson, S.L.; Poirier, S.J.; Grimstad, P.R.; Craig, G.B., Jr. Vector competence of Aedes hendersoni (Diptera: Culicidae) for La Crosse virus: Lack of impaired function in virus-infected salivary glands and enhanced virus transmission by sporozoite-infected mosquitoes. J. Med. Entomol. 1992, 29, 483-488. [CrossRef]

40. Turell, M.J.; Britch, S.C.; Aldridge, R.L.; Kline, D.L.; Boohene, C.; Linthicum, K.J. Potential for mosquitoes (Diptera: Culicidae) from Florida to transmit Rift Valley fever virus. J. Med. Entomol. 2013, 50, 1111-1117. [CrossRef] [PubMed]

41. Gaidamovich, S.Y.; Khutoretskaya, N.V.; Lvova, A.I.; Sveshnikova, N.A. Immunofluorescent staining study of the salivary glands of mosquitoes infected with group A arboviruses. Intervirology 1973, 1, 193-200. [CrossRef] [PubMed]

42. Janzen, H.G.; Rhodes, A.J.; Doane, F.W. Chikungunya virus in salivary glands of Aedes aegypti (L.): An electron microscope study. Can. J. Microbiol. 1970, 16, 581-586. [CrossRef] [PubMed]

43. Salazar, M.I.; Richardson, J.H.; Sanchez-Vargas, I.; Olson, K.E.; Beaty, B.J. Dengue virus type 2: Replication and tropisms in orally infected Aedes aegypti mosquitoes. BMC Microbiol. 2007, 7, 1471-2180. [CrossRef] [PubMed]

44. Coffey, L.L.; Failloux, A.B.; Weaver, S.C. Chikungunya virus-vector interactions. Viruses 2014, 6, 4628-4663. [CrossRef] [PubMed]

45. Kendrick, K.; Stanek, D.; Blackmore, C. Transmission of chikungunya virus in the continental United States-Florida, 2014. MMWR Morb. Mortal. Wkly. 2014, 63, 1137. 